\title{
New dinosaur egg material from Yunxian, Hubei Province, China resolves the classification of dendroolithid eggs
}

Shukang Zhang, Tzu-Ruei Yang, Zhengqi Li, and Yongguo Hu

Acta Palaeontologica Polonica 63 (4), 2018: 671-678 doi:https://doi.org/10.4202/app.00523.2018

The oofamily Dendroolithidae is a distinct group of dinosaur eggs reported from China and Mongolia, which is characterized by branched eggshell units and irregular pore canals. The ootaxonomic inferences, however, were rarely discussed until now. A colonial nesting site was recently uncovered from the Qinglongshan region, Yunxian, Hubei Province, China. More than 30 dendroolithid egg clutches outcrop on the Tumiaoling Hill, including an extremely gigantic clutch containing 77 eggs. All clutches were exposed in the Upper Cretaceous fluvial-deposited Gaogou For mation. In this study, we emend the diagnosis of the oogenus Placoolithus and assign all dendroolithid eggs from the Tumiaoling Hill to a newly emended oospecies Placoolithus tumiaolingensis that shows greatly variable eggshell microstructure. Moreover, our study also disentangles the previous vexing classification of dendroolithid eggs. We conclude that Dendroolithus tumiaolingensis, D. hongzhaiziensis, and Paradendroolithus qinglongshanensis, all of which were previously reported from Yunxian, should be assigned to the newly emended oospecies Placoolithus tumiaolingensis.

Key words: Dendroolithidae, Placoolithus, colonial nesting, Cretaceous, China, Yunxian, Tumiaoling Hill.

Shukang Zhang [zhangshukang@ivpp.ac.cn], Institute of Vertebrate Paleontology and Paleoanthropology, Chinese Academy of Science, 142 Xizhimenwai Street, Beijing, China. Tzu-Ruei Yang [tryang@uni-bonn.de], Steinmann-Institut für Geologie, Mineralogie and Paläontologie, Rheinische-Friedrich-Wilhelms Universitat Bonn, Nussallee 8, Bonn, Germany. Zhengqi Li [972243785@qq.com ], Hubei Institute of Geoscience, 9 Gutian 5th Road, Wuhan, Hubei Province, China. Yongguo Hu [hyg1983916@163.com], Qinglongshan Dinosaur-Egg Fossil Cluster National Geopark, Special 1 Qinglongshan Village, Liubei, Shiyan, Hubei Province, China. 
This is an open-access article distributed under the terms of the Creative Commons

Attribution License (for details please see creativecommons.org), which permits unrestricted use, distribution, and reproduction in any medium, provided the original author and source are credited.

FaF 